\title{
Novel Solid Electrolytes for Li-Ion Batteries: A Perspective from Electron Microscopy Studies
}

\author{
Cheng Ma and Miaofang Chi* \\ Oak Ridge National Laboratory, Center for Nanophase Materials Sciences, Oak Ridge, TN, USA
}

OPEN ACCESS

Edited by:

Shyue Ping Ong,

University of California San Diego,

USA

Reviewed by:

Paulina Pótrolniczak, Institute of Non-Ferrous Metals

Division in Poznań Central Laboratory of Batteries and Cells, Poland Yan E. Wang, MIT, USA

${ }^{*}$ Correspondence: Miaofang Chi chim@ornl.gov

Specialty section:

This article was submitted to Energy Storage,

a section of the journal

Frontiers in Energy Research

Received: 01 March 2016

Accepted: 22 May 2016

Published: 08 June 2016

Citation:

Ma C and Chi M (2016) Novel Solid

Electrolytes for Li-Ion Batteries:

A Perspective from Electron

Microscopy Studies.

Front. Energy Res. 4:23.

doi: 10.3389/fenrg.2016.00023
Solid electrolytes can simultaneously overcome two of the most formidable challenges of Li-ion batteries: the severe safety issues and insufficient energy densities. However, before they can be implemented in actual batteries, the ionic conductivity needs to be improved and the interface with electrodes must be optimized. The prerequisite for addressing these issues is a thorough understanding of the material's behavior at the microscopic and/or the atomic level. (Scanning) transmission electron microscopy is a powerful tool for this purpose, as it can reach an ultrahigh spatial resolution. Here, we review recent electron microscopy investigations on the ion transport behavior in solid electrolytes and their interfaces. Specifically, three aspects will be highlighted: the influence of grain interior atomic configuration on ionic conductivity, the contribution of grain boundaries, and the behavior of solid electrolyte/electrode interfaces. Based on this, the perspectives for future research will be discussed.

Keywords: lithium battery, solid electrolyte, electron microscopy, atomic resolution analysis, interface

\section{INTRODUCTION}

With the exhaustion of fossil fuels, high-performance energy storage devices have received significant attention in recent years (Quartarone and Mustarelli, 2011; Bruce et al., 2012). While the Li-ion battery (LIB) is a very promising alternative power source, the safety concerns and insufficient energy density have hindered its implementation in heavy-duty applications, e.g., electric vehicles and grid energy storage (Quartarone and Mustarelli, 2011; Bruce et al., 2012). Fortunately, these issues can be addressed by integrating novel solid electrolytes (Quartarone and Mustarelli, 2011; Takada, 2013; Wang et al., 2015). On the one hand, these solid materials are typically non-flammable and impossible to leak, which circumvents the safety issues associated with conventional organic liquid electrolytes. This is a prerequisite for large-scale application. On the other hand, the energy density can also be effectively improved. The much larger electrochemical window allows for the use of advanced electrode materials that are incompatible with conventional liquid electrolytes. In addition, by eliminating the need for bulky safety mechanisms, the battery size can be greatly reduced. Because of these advantages, solid electrolytes have received tremendous interest in recent years.

However, two grand challenges must be overcome before solid electrolytes can be used in commercial batteries. First, their ionic conductivity is typically low, preventing a fast charge and discharge (Takada, 2013; Wang et al., 2015). Second, forming a stable conductive interface between the solid electrolyte and electrode is difficult (Zhu et al., 2015 \& 2016; Richards et al., 2016). Overcoming the first challenge requires a mechanistic understanding of the interplay between Li migration and the atomic framework of the material. For the second challenge, the correlation between 
interface structure/chemistry and ionic transport must first be systematically established. Clearly, both tasks demand structural and chemical analysis with an ultrahigh spatial resolution.

Transmission electron microscopy (TEM), most notably aberration-corrected scanning transmission electron microscopy (STEM), is an ideal tool for gaining critical atomic level insight. It is not only capable of directly visualizing the atomic configurations but can also elucidate chemical information at a sub-angstrom spatial resolution using electron energy loss spectroscopy (EELS) and energy-dispersive X-ray spectroscopy (EDS) (Pennycook, 1992; Muller et al., 2008; Chi et al., 2011; Yabuuchi et al., 2011; Wu et al., 2015). However, STEM investigations of solid electrolytes pose numerous challenges as the high Li mobility and poor electronic conductivity make these materials highly vulnerable to electron irradiation damage (Egerton et al., 2004). Fortunately, with the significantly improved capabilities for imaging and TEM specimen preparation, this issue has been greatly alleviated in recent years. Several beam-sensitive materials that could not be studied previously can now be analyzed at the atomic scale (Ma et al., 2015), and many of these studies have made significant contributions toward the research on solid electrolytes.

The present mini-review will highlight electron microscopy investigations for three important factors governing the behavior of solid electrolytes: (1) influence of grain interior atomic configuration on ionic conductivity, (2) impacts of grain boundaries, and (3) behavior of solid electrolyte-electrode interfaces. Based on this, opportunities, challenges, and perspectives for future research will be discussed.

\section{INFLUENCE OF GRAIN INTERIOR ATOMIC CONFIGURATION ON IONIC CONDUCTIVITY}

Li migration within the crystalline lattice is dictated by the atomic framework, which forms channels for Li transport. A precise understanding of the atomic structure is required to explain ionic transport within the lattice. With its ultrahigh spatial resolution and sensitivity to subtle differences in diffraction, (S)TEM not only complements X-ray and neutron scattering studies but also provides unique insights at the atomic level. Recent microscopy studies mainly focused on two systems: $\mathrm{Li}_{7} \mathrm{La}_{3} \mathrm{Zr}_{2} \mathrm{O}_{12}$ (LLZO) and $\mathrm{Li}_{3 x} \mathrm{La}_{2 / 3-x} \mathrm{TiO}_{3}$ (LLTO).

$\mathrm{Li}_{7} \mathrm{La}_{3} \mathrm{Zr}_{2} \mathrm{O}_{12}$ is currently the most promising oxide solid electrolyte due to the coexistence of an excellent stability against Li metal and a relatively high conductivity (Murugan et al., 2007; Cussen, 2010). It crystallizes in the garnet structure with two polymorphs (Cussen, 2010): a cubic phase with a relatively high conductivity (c-LLZO) and a less conductive tetragonal phase (t-LLZO). Distinguishing these two phases is critical to properly interpret the ionic transport behavior. The precession electron diffraction (PED) study by Buschmann et al. (2011) successfully differentiated these two phases by circumventing the influence from double diffraction. This result further confirmed that Al-doping is critical for stabilizing the cubic phase. When combined with neutron diffraction, it was found that the Li sites in c-LLZO, unlike those in t-LLZO, are partially filled. The high concentration of vacancies in c-LLZO gives rise to a higher $\mathrm{Li}$ mobility and superior conductivity. Besides the PED study, Buschmann et al. also tried to perform high-resolution TEM (HRTEM), but detailed analysis was prevented by the electron beam-irradiation damage. Recently, this issue was successfully alleviated by Ma et al. (2015). The careful selection of imaging and specimen preparation conditions enabled a high-quality, atomic resolution (S)TEM/EELS analysis (Figures 1A,B). Ma's research demonstrated that c-LLZO maintains its cubic crystal structure even in an aqueous environment with a $\mathrm{pH}>7$. Such a high structural stability indicates that c-LLZO offers a robust atomic framework for Li transport. Given the high ionic conductivity, Li compatibility, and desirable structural stability against aqueous solutions with a broad range of $\mathrm{pH}$ values, LLZO is a promising candidate for the separator in novel aqueous Li batteries.

Another system that has been extensively interrogated by electron microscopy is LLTO, which has a perovskite-type structure (Stramare et al., 2003). By varying the composition and/or processing conditions, multiple polymorphs with different ionic conductivity may be obtained. Regardless, most of them exhibit an alternate stacking between La-rich and La-poor A-site layers, and Li migration is favored by the La-poor layers. The highest bulk conductivity is $10^{-3} \mathrm{~S} \mathrm{~cm}^{-1}$, approaching that of conventional liquid electrolytes $\left(10^{-2} \mathrm{~S} \mathrm{~cm}^{-1}\right)$ (Takada, 2013). Therefore, an in-depth understanding of the origin for such exceptional performances is crucial for designing highly conductive solid electrolytes. The (S)TEM studies made important contributions toward this cause. Taking advantage of the sensitivity of annularbright-field (ABF) STEM imaging to light elements such as $\mathrm{Li}$, Gao et al. (2013) directly visualized the variation of Li positions in different LLTO polymorphs. Li was observed to reside at the $\mathrm{O} 4$ window for the $\mathrm{Li}$-poor composition $\mathrm{La}_{0.62} \mathrm{Li}_{0.16} \mathrm{TiO}_{3}$, but near the A-site position for the Li-rich composition $\mathrm{La}_{0.56} \mathrm{Li}_{0.33} \mathrm{TiO}_{3}$. The Li content, valence state of cations, and geometry of the oxygen octahedra in the La-rich and La-poor layers were also revealed by EELS. Furthermore, domain structures associated with the ordering between La-rich and La-poor layers were examined (Gao et al., 2014). With La blocking the Li pathways, the domain boundaries were found to impede ionic transport. Beyond this, structural features that cannot be easily detected by diffraction methods may also be visualized. As mentioned above, the Li transport within LLTO relies on the La-poor layers. However, none of the previous diffraction studies detected such crucial features in the most conductive polymorph, the $1350^{\circ} \mathrm{C}$-quenched $\mathrm{La}_{0.56} \mathrm{Li}_{0.33} \mathrm{TiO}_{3}$ (Stramare et al., 2003). As a result, its ionic transport mechanism remained unclear for years. Recently, an atomic resolution STEM study directly visualized the previously overlooked short-range-ordered Li pathways in this material (Ma et al., 2016). The coherence length of the ordering was found to be at the mesoscopic scale (below $10 \mathrm{~nm}$ ), which prevented it from being detected by most diffraction methods. In combination with molecular dynamics (MD) simulations, this observation indicated that such an elusive mesoscopic framework can most effectively maximize the number of Li transport pathways, leading to a high conductivity. The discovery not only reconciled the long-existing structure-property inconsistency but also pointed out a new angle on improving ionic conductivity. 

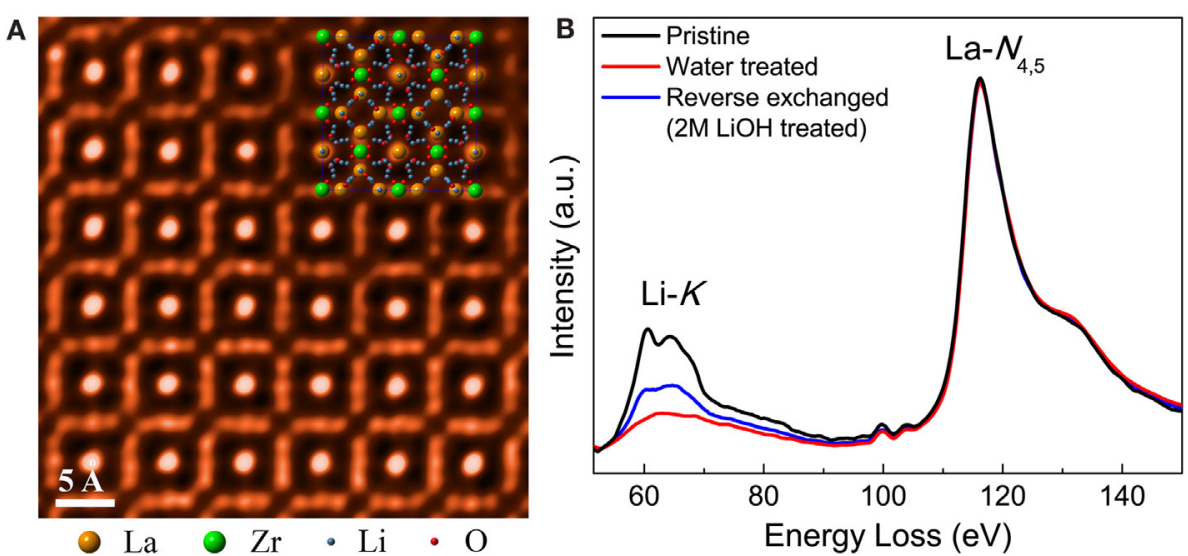

FIGURE 1 | (A) The atomic structure of the electron beam-sensitive solid electrolyte LLZO successfully visualized by high-angle annular dark-field (HAADF) STEM imaging. (B) EELS data of LLZO after the $\mathrm{Li}^{+} / \mathrm{H}^{+}$exchange with different aqueous solutions. The Li content can be precisely monitored. Reproduced with permission from (Ma et al., 2015).

Although atomic resolution (S)TEM greatly benefited the fundamental understanding of ionic transport, current studies are limited to oxides. In comparison, sulfide solid electrolytes, regardless of their higher conductivity (Takada, 2013), are rarely investigated. Microscopy studies on these materials are extremely challenging because of (1) the vulnerability of weak Li-S bonds to electrons and (2) their sensitivity to ambient atmosphere. If these issues can be mitigated, (S)TEM will play an even more critical role in the research of solid electrolytes.

\section{IMPACTS OF GRAIN BOUNDARIES}

Although the research on solid electrolytes primarily focuses on the grain interior, grain boundaries are frequently the actual bottleneck. While the bulk conductivity of many solid electrolytes is already comparable to those of conventional liquid electrolytes, their large grain boundary resistance typically lowers the total conductivity by orders of magnitude (Takada, 2013). Due to the absence of a proper understanding on the grain boundary Li conduction mechanism, a targeted optimization is not yet possible.

Grain boundaries in solids are often confined to a very small length scale with widths of only a few unit cells. Therefore, STEM, with its sub-angstrom resolution, appears to be an ideal tool to study them. Ma et al. (2014) successfully utilized atomic resolution STEM/EELS to unravel the atomic-scale origin of the large grain boundary resistance in LLTO. Most grain boundaries were observed to show darker $Z$-contrast than the adjacent grains, suggesting that the average atomic number at the grain boundary is lower. Further atomic-scale analysis showed that the grain boundary atomic configuration significantly deviated from that of the grain interior (Figures 2A,B). Instead of the $\mathrm{ABO}_{3}$ perovskite structure, such reconstructed grain boundaries are essentially a binary $\mathrm{Ti}-\mathrm{O}$ layer, forbidding the abundance of the charge carrier $\mathrm{Li}^{+}$. Therefore, they act as internal barriers for Li transport. This topic has also been investigated by HRTEM and EDS. In addition, Gellert et al. (2012) studied the grain boundaries in lithium aluminum titanium phosphate (LATP).
Depending on the relative orientation between the neighboring grains, two types of grain boundaries were observed. If the orientations are similar, a thick crystalline grain boundary will be present. Its high degree of crystallinity was believed to allow for a relatively facile ionic transport. If the orientations differ greatly, a thinner but amorphous layer forms, which was believed to be highly resistive.

Unlike the two materials discussed above, LLZO exhibits a grain boundary resistance comparable to that of the grain interior (Murugan et al., 2007). However, the origin of this benign behavior remains unknown. Several research groups have attempted to study LLZO grain boundaries using electron microscopy, but the results are inconsistent. Kumazaki et al. (2011) observed amorphous $\mathrm{Li}-\mathrm{Al}-\mathrm{Si}-\mathrm{O}$ and nanocrystalline $\mathrm{LiAlSiO}_{4}$ at the LLZO grain boundaries. In contrast, clean grain boundaries, which are free of any second phase or compositional variations, were reported by Wolfenstine et al. (2012). Systematic investigations with higher spatial resolution are necessary to reach a conclusive explanation.

These studies demonstrate that the grain boundaries, despite their highly localized nature, can be effectively investigated using (S)TEM combined with local analytical methods, such as EELS and EDS. However, current efforts in this area are very limited. Before a systematic understanding and a rational optimization of Li transport at grain boundaries can be realized, further in-depth investigations are needed.

\section{BEHAVIOR OF ELECTROLYTE- ELECTRODE INTERFACES}

The stable and conductive electrode/electrolyte interface is a prerequisite for the long-term operation of solid electrolytebased batteries (Zhu et al., 2015 \& 2016; Richards et al., 2016). Nevertheless, due to the absence of a mechanistic understanding to guide the rational improvement, it is still very difficult to form such interfaces. As the first step toward this goal, a direct experimental observation of the interfaces is essential. 

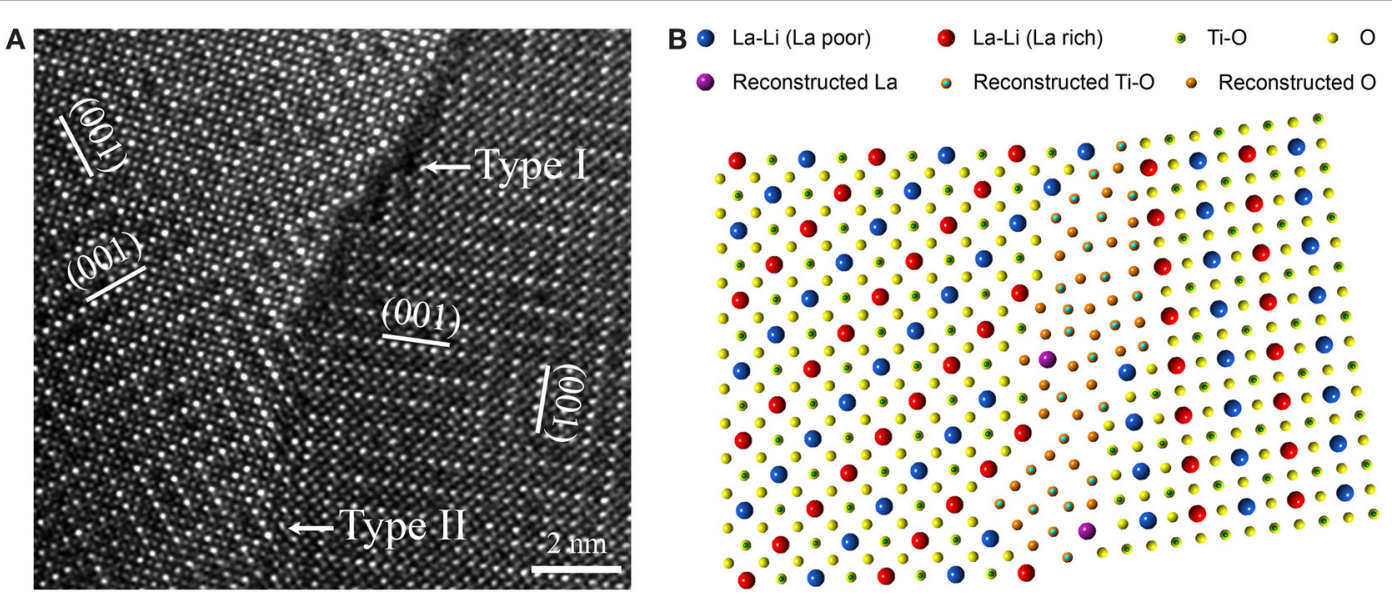

FIGURE 2 | (A) Atomic resolution HAADF-STEM image of a grain boundary in LLTO. (B) Atomic model of the Li-deficient LLTO grain boundary based on a comprehensive STEM/EELS study. Reproduced with permission from (Ma et al., 2014).

Although no atomic resolution electron microscopy studies have been reported to date, interfaces between cathode materials and several solid electrolytes have been examined via nanoelectron diffraction (NED), STEM, and EDS. Kim et al. (2011) investigated the interfacial stability between LLZO and $\mathrm{LiCoO}_{2}$ (LCO). An LCO thin film was grown on the polished surface of the LLZO ceramic through pulsed laser deposition at $937 \mathrm{~K}$. TEM observations revealed the existence of an interface reaction layer of $\sim 50 \mathrm{~nm}$ thick. EDS line profile measurements and NED acquired in the vicinity of the interface suggested that this reaction layer consisted of $\mathrm{La}_{2} \mathrm{CoO}_{4}$, which is believed to hinder $\mathrm{Li}$ diffusion. In addition, the interface between $\mathrm{LCO}$ and $\mathrm{Li}_{2} \mathrm{~S}-\mathrm{P}_{2} \mathrm{~S}_{5}$, a prototypical sulfide electrolyte, was studied by Sakuda et al. (2009). The interface was simply formed by mechanical grinding. After charging, an interfacial layer associated with the mutual diffusion of $\mathrm{Co}, \mathrm{P}$, and $\mathrm{S}$ emerged, and this layer gave rise to a large resistance. A similar behavior was observed between $\mathrm{LiMn}_{2} \mathrm{O}_{4}$ and $\mathrm{Li}_{2} \mathrm{~S}_{-} \mathrm{P}_{2} \mathrm{~S}_{5}$ (Kitaura et al., 2010). An interfacial layer resulting from the diffusion of $\mathrm{Mn}$ into the solid electrolyte was observed and believed to yield large resistance. These electron microscopy studies suggest that a reaction layer may frequently form between the solid electrolyte and cathode due to inter-diffusion. Unlike the solid electrolyte interface (SEI) in conventional LIBs, reaction layers at solid electrolyte/ electrode interfaces are usually detrimental rather than beneficial, as they typically impede the ionic transport (Qian et al., 2015).

Beyond these experimentally observed reaction layers, highly localized interfacial decomposition at the solid electrolyte and electrode interfaces was frequently speculated, although they show a certain degree of stability in electrochemical measurements (Zhu et al., 2015 \& 2016; Richards et al., 2016). However, most of such speculations are proposed based on theoretical calculations. The experimental verification is quite challenging due to the extremely small length scale of the speculated thickness and the high volatility/instability of Li metal (Wenzel et al., 2015, 2016). (S)TEM, which can probe local features at an extremely high spatial resolution down to sub-angstrom level, presents excellent opportunities to interrogate these intriguing interfacial behaviors.

\section{SUMMARY AND PROSPECTS}

In this mini-review, we discussed recent progress of (S)TEM studies on solid electrolytes for Li batteries. With the success in alleviating the challenges caused by electron beam irradiation damage, more and more investigations that could not be performed previously are reported. These studies clarified several long-standing confusions regarding the structure-property relationship, provided first experimental insights into the large grain boundary resistance, and contributed insights regarding the reaction layer at cathode/SEIs.

Regardless, further challenges remain. For the ionic transport within the grain, sulfide electrolytes, which often exhibit a higher conductivity than oxides, demand a thorough study at atomic scale. Their vulnerary to electron beam, due to the weak bonds of $\mathrm{Li}$ with $\mathrm{S}$ in the structure and limited electron conductivity, significantly limit reliable measurements of their atomic and electronic structures in TEMs. In order to understand the role of grain boundaries in solid electrolytes, a wide range of materials need to be investigated in order to establish the systematic understanding. In particular, the materials with benign grain boundaries deserve special attention, as they may inspire the design of materials with conductive grain boundaries. For the solid electrolyte/electrode interface, one of the most pressing tasks is to verify the highly localized interfacial reaction layers that are proposed recently by theoretical works. Furthermore, the variation of these interfaces with composition, processing conditions, and cycling also remained to be investigated. It must be emphasized that recently developed in situ TEM techniques, such as in situ heating, and in situ electrochemical cycling with a desirable spatial resolution, will greatly facilitate these studies (Gu et al., 2013; Chi et al., 2015; Zeng et al., 2015). Their capability of real-time, high-resolution structural/chemical analysis will provide unique insights that cannot be acquired otherwise. With the recent remarkable developments in microscopy instrumentations, such as fast cameras and detectors, low voltage TEMs, and multi-functional specimen stages, these challenges should be 
overcome in the near future, and electron microscopy is expected to play an increasingly significant role in the research of Li-ionconducting solid electrolytes.

\section{AUTHOR CONTRIBUTIONS}

All authors listed have made substantial, direct, and intellectual contribution to the work and approved it for publication.

\section{REFERENCES}

Bruce, P. G., Freunberger, S. A., Hardwick, L. J., and Tarascon, J.-M. (2012). Li-O and Li-S batteries with high energy storage. Nat. Mater. 11, 19-29. doi:10.1038/ nmat3191

Buschmann, H., Dolle, J., Berendts, S., Kuhn, A., Bottke, P., Wilkening, M., et al. (2011). Structure and dynamics of the fast lithium ion conductor " $\mathrm{Li}_{7} \mathrm{La}_{3} \mathrm{Zr}_{2} \mathrm{O}_{12}$ ". Phys. Chem. Chem. Phys. 13, 19378-19392. doi:10.1039/c1cp22108f

Chi, M. F., Mizoguchi, T., Martin, L. W., Bradley, J. P., Ikeno, H., Ramesh, R., et al. (2011). Atomic and electronic structures of the $\mathrm{SrVO}_{3}-\mathrm{LaAlO}_{3}$ interface. J. Appl. Phys. 110, 046104. doi:10.1063/1.3601870

Chi, M. F., Wang, C., Lei, Y. K., Wang, G. F., Li, D. G., More, K. L., et al. (2015). Surface faceting and elemental diffusion behaviour at atomic scale for alloy nanoparticles during in situ annealing. Nat. Commun. 6, 8925. doi:10.1038/ncomms9925

Cussen, E. J. (2010). Structure and ionic conductivity in lithium garnets. J. Mater. Chem. 20, 5167-5173. doi:10.1039/b925553b

Egerton, R. F., Li, P., and Malac, M. (2004). Radiation damage in the TEM and SEM. Micron 35, 399-409. doi:10.1016/j.micron.2004.02.003

Gao, X., Fisher, C. A. J., Kimura, T., Ikuhara, Y. H., Kuwabara, A., Moriwake, H., et al. (2014). Domain boundary structures in lanthanum lithium titanates. J. Mater. Chem. A 2, 843-852. doi:10.1039/C3TA13726K

Gao, X., Fisher, C. A. J., Kimura, T., Ikuhara, Y. H., Moriwake, H., Kuwabara, A., et al. (2013). Lithium atom and A-site vacancy distributions in lanthanum lithium titanate. Chem. Mater. 25, 1607-1614. doi:10.1021/cm3041357

Gellert, M., Gries, K. I., Yada, C., Rosciano, F., Volz, K., and Roling, B. (2012). Grain boundaries in a lithium aluminum titanium phosphate-type fast lithium ion conducting glass ceramic: microstructure and nonlinear ion transport properties. J. Phys. Chem. C 116, 22675-22678. doi:10.1021/jp305309r

Gu, M., Parent, L. R., Mehdi, B. L., Unocic, R. R., McDowell, M. T., Sacci, R. L., et al. (2013). Demonstration of an electrochemical liquid cell for operando transmission electron microscopy observation of the lithiation/delithiation behavior of Si nanowire battery anodes. Nano Lett. 13, 6106-6112. doi:10.1021/nl403402q

Kim, K. H., Iriyama, Y., Yamamoto, K., Kumazaki, S., Asaka, T., Tanabe, K., et al. (2011). Characterization of the interface between $\mathrm{LiCoO}_{2}$ and $\mathrm{Li}_{7} \mathrm{La}_{3} \mathrm{Zr}_{2} \mathrm{O}_{12}$ in an all-solid-state rechargeable lithium battery. J. Power Sources 196, 764-767. doi:10.1016/j.jpowsour.2010.07.073

Kitaura, H., Hayashi, A., Tadanaga, K., and Tatsumisago, M. (2010). All-solidstate lithium secondary batteries using $\mathrm{LiMn}_{2} \mathrm{O}_{4}$ electrode and $\mathrm{Li}_{2} \mathrm{~S}-\mathrm{P}_{2} \mathrm{~S}_{5}$ solid electrolyte. J. Electrochem. Soc. 157, A407-A411. doi:10.1149/1.3298441

Kumazaki, S., Iriyama, Y., Kim, K.-H., Murugan, R., Tanabe, K., Yamamoto, K., et al. (2011). High lithium ion conductive $\mathrm{Li}_{7} \mathrm{La}_{3} \mathrm{Zr}_{2} \mathrm{O}_{12}$ by inclusion of both $\mathrm{Al}$ and Si. Electrochem. Commun. 13, 509-512. doi:10.1016/j.elecom.2011.02.035

Ma, C., Chen, K., Liang, C. D., Nan, C. W., Ishikawa, R., More, K., et al. (2014). Atomic-scale origin of the large grain-boundary resistance in perovskite Li-ionconducting solid electrolytes. Energy Environ. Sci. 7, 1638-1642. doi:10.1039/ c4ee $00382 \mathrm{a}$

Ma, C., Cheng, Y., Chen, K., Li, J., Sumpter, B., Nan, C.-W., et al. (2016). Mesoscopic framework enables facile ionic transport in solid electrolytes for Li batteries. Adv. Energy Mater. doi:10.1002/aenm.201600053

Ma, C., Rangasamy, E., Liang, C. D., Sakamoto, J., More, K. L., and Chi, M. F. (2015). Excellent stability of a lithium-ion-conducting solid electrolyte upon reversible $\mathrm{Li}^{+} / \mathrm{H}^{+}$exchange in aqueous solutions. Angew. Chem. Int. Ed. 54, 129-133. doi:10.1002/anie.201410930

Muller, D. A., Kourkoutis, L. F., Murfitt, M., Song, J. H., Hwang, H. Y., Silcox, J., et al. (2008). Atomic-scale chemical imaging of composition and bonding

\section{FUNDING}

This work was sponsored by the U.S. Department of Energy (DOE), Office of Science, Office of Basic Energy Sciences, Materials Sciences and Engineering Division. Materials characterization was performed as part of a user proposal at the Center for Nanophase Materials Sciences, which is a U.S. DOE Office of Science User Facility.

by aberration-corrected microscopy. Science 319, 1073-1076. doi:10.1126/ science. 1148820

Murugan, R., Thangadurai, V., and Weppner, W. (2007). Fast lithium ion conduction in garnet-type $\mathrm{Li}_{7} \mathrm{La}_{3} \mathrm{Zr}_{2} \mathrm{O}_{12}$. Angew. Chem. Int. Ed. 46, 7778-7781. doi:10.1002/anie.200701144

Pennycook, S. J. (1992). Z-contrast transmission electron microscopy - direct atomic imaging of materials. Ann. Rev. Mater. Sci. 22, 171-195. doi:10.1146/ annurev.ms.22.080192.001131

Qian, D., Ma, C., More, K. L., Meng, Y. S., and Chi, M. (2015). Advanced analytical electron microscopy for lithium-ion batteries. NPG Asia Mater. 7, e193. doi:10.1038/am.2015.50

Quartarone, E., and Mustarelli, P. (2011). Electrolytes for solid-state lithium rechargeable batteries: recent advances and perspectives. Chem. Soc. Rev. 40, 2525-2540. doi:10.1039/c0cs00081g

Richards, W. D., Miara, L. J., Wang, Y., Kim, J. C., and Ceder, G. (2016). Interface stability in solid-state batteries. Chem. Mater. 28, 266-273. doi:10.1021/acs. chemmater.5b04082

Sakuda, A., Hayashi, A., and Tatsumisago, M. (2009). Interfacial observation between $\mathrm{LiCoO}_{2}$ electrode and $\mathrm{Li}_{2} \mathrm{~S}-\mathrm{P}_{2} \mathrm{~S}_{5}$ solid electrolytes of all-solid-state lithium secondary batteries using transmission electron microscopy. Chem. Mater. 22, 949-956. doi:10.1021/cm901819c

Stramare, S., Thangadurai, V., and Weppner, W. (2003). Lithium lanthanum titanates: a review. Chem. Mater. 15, 3974-3990. doi:10.1021/cm0300516

Takada, K. (2013). Progress and prospective of solid-state lithium batteries. Acta Mater. 61, 759-770. doi:10.1016/j.actamat.2012.10.034

Wang, Y., Richards, W. D., Ong, S. P., Miara, L. J., Kim, J. C., Mo, Y., et al. (2015). Design principles for solid-state lithium superionic conductors. Nat. Mater. 14, 1026-1031. doi:10.1038/nmat4369

Wenzel, S., Leichtweiss, T., Krüger, D., Sann, J., and Janek, J. (2015). Interphase formation on lithium solid electrolytes - an in situ approach to study interfacial reactions by photoelectron spectroscopy. Solid State Ionics 278, 98-105. doi:10.1016/j.ssi.2015.06.001

Wenzel, S., Weber, D. A., Leichtweiss, T., Busche, M. R., Sann, J., and Janek, J. (2016). Interphase formation and degradation of charge transfer kinetics between a lithium metal anode and highly crystalline $\mathrm{Li}_{7} \mathrm{P}_{3} \mathrm{~S}_{11}$ solid electrolyte. Solid State Ionics 286, 24-33. doi:10.1016/j.ssi.2015.11.034

Wolfenstine, J., Sakamoto, J., and Allen, J. L. (2012). Electron microscopy characterization of hot-pressed $\mathrm{Al}$ substituted $\mathrm{Li}_{7} \mathrm{La}_{3} \mathrm{Zr}_{2} \mathrm{O}_{12}$. J. Sci. Mater. 47, 4428-4431. doi:10.1007/s10853-012-6300-y

Wu, Y., Ma, C., Yang, J. H., Li, Z. C., Allard, L. F., Liang, C. D., et al. (2015). Probing the initiation of voltage decay in Li-rich layered cathode materials at the atomic scale. J. Mater. Chem. A 3, 5385-5391. doi:10.1039/ C4TA06856D

Yabuuchi, N., Yoshii, K., Myung, S. T., Nakai, I., and Komaba, S. (2011). Detailed studies of a high-capacity electrode material for rechargeable batteries, $\mathrm{Li}_{2} \mathrm{MnO}_{3}-\mathrm{LiCo}_{1} / 3 \mathrm{Ni}_{1} / 3 \mathrm{Mn}_{1} / 3 \mathrm{O}_{2}$. J. Am. Chem. Soc. 133, 4404-4419. doi:10.1021/ja108588y

Zeng, Z. Y., Zhang, X. W., Bustillo, K., Niu, K. Y., Gammer, C., Xu, J., et al. (2015). In situ study of lithiation and delithiation of $\mathrm{MoS}_{2}$ nanosheets using electrochemical liquid cell transmission electron microscopy. Nano Lett. 15, 5214-5220. doi:10.1021/acs.nanolett.5b02483

Zhu, Y., He, X., and Mo, Y. (2015). Origin of outstanding stability in the lithium solid electrolyte materials: insights from thermodynamic analyses based on first-principles calculations. ACS Appl. Mater. Interfaces 7, 23685-23693. doi:10.1021/acsami.5b07517 
Zhu, Y., He, X., and Mo, Y. (2016). First principles study on electrochemical and chemical stability of the solid electrolyte-electrode interfaces in all-solid-state Li-ion batteries. J. Mater. Chem. A. 4, 3253-3266. doi:10.1039/C5TA08574H

Conflict of Interest Statement: The authors declare that the research was conducted in the absence of any commercial or financial relationships that could be construed as a potential conflict of interest.
Copyright (c) $2016 \mathrm{Ma}$ and Chi. This is an open-access article distributed under the terms of the Creative Commons Attribution License (CC BY). The use, distribution or reproduction in other forums is permitted, provided the original author(s) or licensor are credited and that the original publication in this journal is cited, in accordance with accepted academic practice. No use, distribution or reproduction is permitted which does not comply with these terms. 